\title{
3D multiphysics modeling of superconducting cavities with a massively parallel simulation suite
}

\author{
Oleksiy Kononenko, Chris Adolphsen, Zenghai Li, Cho-Kuen Ng, and Claudio Rivetta \\ SLAC National Accelerator Laboratory, Menlo Park, California 94025, USA
}

(Received 29 June 2017; published 10 October 2017)

\begin{abstract}
Radiofrequency cavities based on superconducting technology are widely used in particle accelerators for various applications. The cavities usually have high quality factors and hence narrow bandwidths, so the field stability is sensitive to detuning from the Lorentz force and external loads, including vibrations and helium pressure variations. If not properly controlled, the detuning can result in a serious performance degradation of a superconducting accelerator, so an understanding of the underlying detuning mechanisms can be very helpful. Recent advances in the simulation suite ACE3P have enabled realistic multiphysics characterization of such complex accelerator systems on supercomputers. In this paper, we present the new capabilities in ACE3P for large-scale 3D multiphysics modeling of superconducting cavities, in particular, a parallel eigensolver for determining mechanical resonances, a parallel harmonic response solver to calculate the response of a cavity to external vibrations, and a numerical procedure to decompose mechanical loads, such as from the Lorentz force or piezoactuators, into the corresponding mechanical modes. These capabilities have been used to do an extensive rf-mechanical analysis of dressed TESLA-type superconducting cavities. The simulation results and their implications for the operational stability of the Linac Coherent Light Source-II are discussed.
\end{abstract}

DOI: 10.1103/PhysRevAccelBeams.20.102001

\section{INTRODUCTION}

Superconducting radiofrequency (SRF) cavities $[1,2]$ are widely used to accelerate and deflect particle beams for various applications. For example, the $1.3 \mathrm{GHz}$ TESLAlike accelerator cavities can support extremely high external quality factors $\left(\mathrm{Q}_{\mathrm{ext}}\right)$, up to $10^{10}$, but to be practical, they are typically operated with $\mathrm{Q}_{\text {ext }}$ values below $10^{8}$. Still, the rf bandwidth is very narrow, which makes the field stability sensitive to the Lorentz force and various external loads: helium pressure fluctuations and vibrations from $\mathrm{He}$ flow, ground motion and nearby equipment. The forces can lead to significant detuning of the cavities, called microphonics in the case of the loads, which decreases the acceleration gradient if no mitigations are taken.

To compensate for the detuning, the rf power is typically increased to maintain the same gradient. In addition, electromechanical and piezoactuators are used to keep the cavity in tune by essentially controlling their length $[3,4]$. However, due to the excitation of cavity mechanical resonances, the cavity control system design can become fairly complicated $[5,6]$. The design is aided by an understanding of the electromechanical interactions in the cavity

Oleksiy.Kononenko@slac.stanford.edu

Published by the American Physical Society under the terms of the Creative Commons Attribution 4.0 International license. Further distribution of this work must maintain attribution to the author(s) and the published article's title, journal citation, and DOI. and how the rf fields and external loads distort the cavity shape. Even though the mathematical formulation for such a study is relatively straightforward and has been available for quite some time [7], it is still challenging to determine all the involved model parameters with the precision required.

The electromechanical cavity model developed in [8] and later advanced in [9] is based on the ordinary differential equation for a damped harmonic oscillator that is driven by internal and external forces. In this model, the surface displacements of the cavity walls are expanded in terms of mechanical mode excitations and the corresponding change in the resonant frequency is computed by using the Slater perturbation technique [10]. For this approach to provide accurate results, the number of modes required in the expansion needs to be determined as does the coupling of the forces to the mechanical eigenmodes.

Experimental, theoretical and computational efforts have been used to understand this phenomenon [11-16]. In particular, cavity measurements [14-16] can be done to determine the Lorentz force detuning coefficient, the electromechanical transfer function, the mechanical resonant frequencies and damping rates. If the mechanical frequency spectrum is dense or the detuning spectrum changes in time, experimental characterization of the SRF system becomes very complicated.

Commercial simulation tools [17-19] are capable of modeling some of the relevant electromechanical effects in the cavity. However, when the problem size becomes large (as in the case of a dressed cavity including the tuner and 
He vessel), most of the simulation tools designed for conventional desktop computers are inefficient due to computational speed and memory size.

The 3D massively parallel ACE3P simulation suite [20] can be used to overcome these limitations through high performance computing on state-of-the-art supercomputers. ACE3P includes parallel electromagnetic, thermal and mechanical modules for the integrated analysis of accelerator problems at large scale, which are readily applicable to SRF cavities and fully dressed cavities in cryomodules [21-24]. By taking advantage of the existing finite-element framework in ACE3P, we have developed a parallel mechanical eigensolver to determine mechanical resonant frequencies and mode patterns, a parallel harmonic response solver to calculate the system response to external loads, and an analysis tool to calculate the coupling of Lorentz and external forces to the mechanical modes. Furthermore, a simulation workflow has been designed to facilitate coupled electromechanical calculations for largescale problems on supercomputers.

This work was done in support of the SLAC Linac Coherent Light Source-II (LCLS-II) [25] project in which a $4 \mathrm{GeV} c w$ superconducting linac will drive an $\mathrm{x}$-ray free electron laser. The linac includes 35 cryomodules each containing eight TESLA-style, $1.3 \mathrm{GHz}$, niobium cavities [26]. The cavity bandwidth is $32 \mathrm{~Hz}$ and the goal is to keep the peak detuning below $10 \mathrm{~Hz}$ so as to limit the rf power required to compensate it.

The paper is organized as follows. Section II discusses the model [7-9] that provides a mathematical background for the electromechanical interactions in a superconducting cavity. Section III introduces the parallel ACE3P mechanical eigenmode solver and presents the results for the rf characterization of cavity structural resonances. Section IV presents the cavity response to a static Lorentz force and tuner load as well as their coupling to the mechanical eigenmodes. Section V introduces the parallel ACE3P harmonic response solver and compares tuner-to-cavity transfer function predictions with data. Finally, Sec. VI presents the conclusions.

\section{ELECTROMECHANICAL INTERACTIONS IN THE SRF CAVITY}

\section{A. Dynamic electromechanical cavity model}

Given the physical dimensions and thermal design constraints of an SRF cavity, the mechanical dynamics are strongly coupled to the electromagnetic characteristics. For a cavity operating in an accelerator where feedback control is used to stabilize the magnitude and phase of the acceleration field, a full model of the SRF cavity is helpful to design the low-level rf system (LLRF). This model has to include as input variables the rf power to drive the SRF cavity and the forces contributing to the deformation of the cavity.
The forces exerted on the cavity, whether from the tuner or vibrations, change the cavity shape and hence the cavity resonant frequency. By decomposing the shape changes into a sum of mechanical mode excitations, one can compute how time dependent forces couple to these modes, and by determining the detuning that each mode causes, the net detuning of the cavity. The calculations are particularly useful when designing resonance control feedback algorithms so as to avoid exciting mechanical modes (e.g., adjusting the gain to be less than unity when the feedback is positive).

Similar to the formalism in [8], in this paper the displacement of the cavity wall, $\vec{u}(x, y, z, t)$, and the force acting on it, $\vec{F}(x, y, z, t)$, are expanded into a complete infinite set of orthonormal mechanical eigenmodes, $\vec{u}_{\mu}(x, y, z) e^{i \Omega_{\mu} t}$, as

$$
\begin{aligned}
& \vec{u}(x, y, z, t)=\sum_{\mu=1}^{\infty} q_{\mu}(t) \vec{u}_{\mu}(x, y, z), \\
& \vec{F}(x, y, z, t)=\sum_{\mu=1}^{\infty} F_{\mu}(t) \vec{u}_{\mu}(x, y, z),
\end{aligned}
$$

where $q_{\mu}(t)=q_{\mu}^{0} e^{i \Omega_{\mu} t}, F_{\mu}(t)=F_{\mu}^{0} e^{i \Omega_{\mu} t}, q_{\mu}^{0}$ and $F_{\mu}^{0}$ are the corresponding amplitudes, $\Omega_{\mu}=2 \pi \Phi_{\mu}$ is the angular frequency and $\Phi_{\mu}$ the frequency of the mechanical mode $\mu$.

Based on the Lagrange formulation, the equation of motion for a particular mechanical mode $\mu$ becomes

$$
\ddot{q}_{\mu}(t)+\frac{2}{\tau_{\mu}} \dot{q}_{\mu}(t)+\Omega_{\mu}^{2} q_{\mu}(t)=\frac{\Omega_{\mu}^{2}}{c_{\mu}} F_{\mu}(t),
$$

where $\tau_{\mu}$ is the decay time and $c_{\mu}$ is the elastic constant of the mechanical mode $\mu[8,9]$.

$\vec{F}(x, y, z, t)$ is a superposition of the forces acting on the cavity. It can have either a deterministic nature such as the Lorentz force, which is proportional to the cavity gradient squared, or be stochastic and caused, for instance, by coupled mechanical vibrations or turbulence of the liquid helium in a cryomodule.

From Slater's perturbation theory [10] it follows that the angular rf frequency shift $\Delta \omega_{\mu}(\mathrm{t})$ due to a particular mechanical mode $\mu$ is proportional to $q_{\mu}(t)$, so that Eq. (3) can be rewritten as

$$
\begin{aligned}
& \Delta \ddot{\omega}_{\mu}(t)+\frac{2}{\tau_{\mu}} \Delta \dot{\omega}_{\mu}(t)+\Omega_{\mu}^{2} \Delta \omega_{\mu}(t) \\
& \quad=k_{\mu} \Omega_{\mu}^{2} G(t)^{2}+n_{\mu} \Omega_{\mu}^{2} N(t)+m_{\mu} \Omega_{\mu}^{2} M(t),
\end{aligned}
$$

where $\omega_{\mu}(t)=2 \pi f_{\mu}(\mathrm{t})$ and $f_{\mu}(t)$ is the resonant frequency. The Lorentz coefficient $k_{\mu}$ is the frequency shift per unit of gradient $G(t)$ squared, $n_{\mu}$ is the frequency shift per unit of 
axial force $N(t)$ applied by the piezotuner and $m_{\mu}$ is the frequency shift per unit of external force $M(t)$ of a given type (e.g., vibration of the He vessel). The total rf frequency shift, $\Delta \omega(t)$, can be calculated as a sum of the mode contributions as

$$
\Delta \omega(t)=\sum_{\mu=1}^{\infty} \Delta \omega_{\mu}(t)
$$

\section{B. Static electromechanical cavity model}

In the static case, when the external forces are constant, the spatial amplitudes $q_{\mu}^{0}$ and $F_{\mu}^{0}$ from (1) and (2) can be expressed as

$$
\begin{aligned}
& q_{\mu}^{0}=\int_{V_{S}} \vec{u}(x, y, z) \vec{u}_{\mu}(x, y, z) d V_{S}, \\
& F_{\mu}^{0}=\int_{V_{S}} \vec{F}(x, y, z) \vec{u}_{\mu}(x, y, z) d V_{S} .
\end{aligned}
$$

In some applications, the cavity wall can be considered infinitely thin, but with nonuniform wall thicknesses, a finite wall thickness model is needed. Also, the force $\vec{F}$ may act on the cavity surface or on the overall cavity structural volume in the form of thermal stresses or gravity, so a full $3 \mathrm{D}$ representation is required. Therefore, in contrast to [7-9], the integrals in (6) and (7) are taken over $V_{S}$, the $3 \mathrm{D}$ cavity structural volume, rather than just over the two-dimensional surface.

Simplifying Eq. (5), the total static cavity frequency shift can be expressed as

$$
\Delta \omega_{0}=\sum_{\mu=1}^{\infty} \Delta \omega_{0 \mu}
$$

and $\Delta \omega_{0 \mu}$, the static frequency shift due to the eigenmode $\mu$ becomes

$$
\Delta \omega_{0 \mu}=k_{\mu} G_{0}^{2}+n_{\mu} N_{0}+m_{\mu} M_{0},
$$

where $G_{0}, N_{0}$ and $M_{0}$ are the constant gradient, piezotuner and external loads respectively. The static coefficients $k, n$ and $m$ are then defined as follows:

$$
k=\sum_{\mu=1}^{\infty} k_{\mu}, \quad n=\sum_{\mu=1}^{\infty} n_{\mu}, \quad m=\sum_{\mu=1}^{\infty} m_{\mu} .
$$

The static frequency shift for eigenmode $\mu$ can also be determined directly from the fields using Slater's perturbation technique [7],

$$
\frac{\Delta \omega_{0 \mu}}{\omega_{0}}=\frac{\int_{\Delta V_{R F}^{\mu}}\left[\mu_{0}|\vec{H}(x, y, z)|^{2}-\varepsilon_{0}|\vec{E}(x, y, z)|^{2}\right] d V_{R F}}{\left.\left.\int_{V_{R F}^{0}}\left[\mu_{0}|\vec{H}(x, y, z)|^{2}+\varepsilon_{0} \mid \vec{E} x, y, z\right)\right|^{2}\right] d V_{R F}},
$$

where $V_{R F}^{0}$ is the unperturbed internal volume of the cavity, $\Delta V_{R F}^{\mu}$ is the volume perturbation, $\varepsilon_{0}$ is the vacuum permittivity, $\mu_{0}$ is the vacuum permeability, and $\vec{E}$ and $\vec{H}$ are the electric and magnetic field amplitudes of the corresponding cavity rf mode. Hence, the coupling coefficients $k_{\mu}, n_{\mu}$ or $m_{\mu}$ are easily calculated from (9) by considering each perturbation individually.

These coefficients can be used in the dynamic equation (4) to solve for the frequency variation $\Delta \omega_{\mu}(\mathrm{t})$ as a response to a specific force term $a_{\mu} e^{i \Omega t}$ :

$\Delta \ddot{\omega}_{\mu}(t)+2 \zeta_{\mu} \Omega_{\mu} \Delta \dot{\omega}_{\mu}(t)+\Omega_{\mu}^{2} \Delta \omega_{\mu}(t)=a_{\mu} \Omega_{\mu}^{2} e^{i \Omega t}$,

where $\zeta_{\mu}$ is the mode damping ratio defined as $\frac{1}{\tau_{\mu \Omega_{\mu}}}, a_{\mu}$ and $\Omega$ are the coupling coefficient and the angular frequency, respectively. The steady-state solution to Eq. (12) is fairly straightforward [27]:

$$
\Delta \omega_{\mu}(t)=\frac{a_{\mu} e^{i(\Omega t+\phi)}}{\sqrt{\left(\Omega_{\mu}^{2}-\Omega^{2}\right)^{2}+\left(2 \zeta_{\mu} \Omega \Omega_{\mu}\right)^{2}}},
$$

where the phase $\phi$ is

$$
\phi=\arctan \left(\frac{2 \zeta_{\mu} \Omega \Omega_{\mu}}{\Omega^{2}-\Omega_{\mu}^{2}}\right) .
$$

Although this formulation is well known, it is difficult to accurately compute the coupling coefficients $q_{\mu}, k_{\mu}, n_{\mu}$ and $m_{\mu}$ using conventional computational techniques. Also, in this study it is not clear how to determine $\zeta_{\mu}$, the damping factors for the mechanical modes [28].

\section{MECHANICAL EIGENMODES OF THE LCLS-II SRF CAVITY}

\section{A. Finite element formulation}

To use the formalism discussed above requires determining the mechanical eigenmodes of the cavity and their contribution to its detuning. For this purpose, the following equations governing the linear elasticity [29] of the materials involved were used:

$$
\begin{gathered}
\nabla \cdot \sigma+\vec{F}=\rho \ddot{\vec{u}}, \\
\varepsilon=\frac{1}{2}\left(\nabla \vec{u}+\nabla \vec{u}^{T}\right), \\
\sigma=C \varepsilon,
\end{gathered}
$$

where $\sigma$ and $\varepsilon$ are the stress and strain tensors, $\vec{u}$ is an unknown displacement vector, $\vec{F}$ is the vector of the 
external forces, $C$ is the stiffness tensor and $\rho$ is the material density, respectively. These equations hold true in a 3D domain $V_{S}$ under the linearization assumptions: infinitesimal strains and linear relationships between the components of the stress and strain.

The equation of motion, Eq. (15), the straindisplacement equation, Eq. (16), and Hook's law, Eq. (17), can be simplified to the weak form of the problem in a standard way [30]:

$$
\int_{V_{S}}\left[\mu\left(\nabla \vec{u}+\nabla \vec{u}^{T}\right)+\lambda(\nabla \cdot \vec{u}) I\right] \cdot \nabla \vec{v} d V_{S}=\Omega^{2} \int_{V_{S}} \rho(\vec{u} \cdot \vec{v}) d V_{S},
$$

where $\lambda$ and $\mu$ are the material-dependent Lamé coefficients, $\vec{v}$ is a test function. Eq. (18) is subject to the boundary conditions:

$$
\begin{gathered}
\vec{u}=\vec{u}_{0} \quad \text { on } \quad \Gamma_{D}, \\
\sigma \cdot \vec{n}=\vec{t}_{0} \quad \text { on } \quad \Gamma_{N},
\end{gathered}
$$

where $\vec{u}_{0}$ is the imposed displacement on the Dirichlet boundary $\Gamma_{D}$, and $\vec{t}_{0}$ is the imposed pressure on the Neumann boundary $\Gamma_{N}$. To study the natural cavity vibrations we consider only homogenous boundary conditions, i.e., $\vec{u}_{0}=0$ if the surface is fixed and $\vec{t}_{0}=0$ if the surface is free.

Using the finite element method (FEM) [29], Eqs. (18)-(20) are discretized as

$$
K \hat{u}=\hat{\Omega}^{2} M \hat{u}
$$

where $K$ and $M$ are the stiffness and mass matrices, respectively, $\hat{u}$ is a vector of the basis coefficients for the displacements, $\hat{\Omega}$ is an angular frequency approximation.

Based on the above formulation, a massively parallel mechanical eigenmode solver was developed within the C++/MPI framework of the ACE3P simulation suite [30].
This solver is capable of performing an accurate 3D characterization of the mechanical modes on supercomputers [31], taking into account complicated geometrical details of the SRF structures.

\section{B. Structural model of the LCLS-II cavity}

A fully dressed $1.3 \mathrm{GHz}$ LCLS-II cavity is shown in Fig. 1. Eight such cavities are mounted in a cryomodule with connecting bellows. A simplified dressed cavity model is shown in Fig. 2 that does not include the power coupler nor the two-phase He feed line, which only weakly constrains the He vessel motion due to the associated bellows. The coupling to the neighboring cavities is also ignored for the same reason. For this model, the tuner assembly [4] is greatly simplified to one with an equivalent spring constant. Otherwise, the simulation model takes into account the full 3D geometry of the cavity (with stiffening rings) in a helium vessel including the bellows and conical connections between the vessel and the beam pipe. However, the effect of the superfluid He that fills the He vessel is not considered.

As for the boundary conditions, an important issue is the extent to which the He vessel motion is constrained by the external support system as this has a significant effect on the eigenfrequencies. The vessel is supported on four horizontal tabs at its midplane (two tabs are shown in Figs. 1 and 2) that we assume to be fixed in the $Y$ direction, as gravity will prevent the massive structure from moving vertically. The tabs fit into a $\mathrm{C}$-shaped assembly with spring-loaded roller-bearing contacts. These assemblies allow for axial motion that is driven by the thermal contraction occurring during the cooldown of the cryomodule. For this study we consider two extremes: where the vessel is free to move axially and where its axial motion is fixed due to frictional forces.

Another complication is an invar rod that runs the length of the cryomodule and has a contact connection to each cavity through a cylindrical tab at the top of the He vessel (see Fig. 1). This contact will also constrain the vessel motion axially, at least in one direction, and we consider

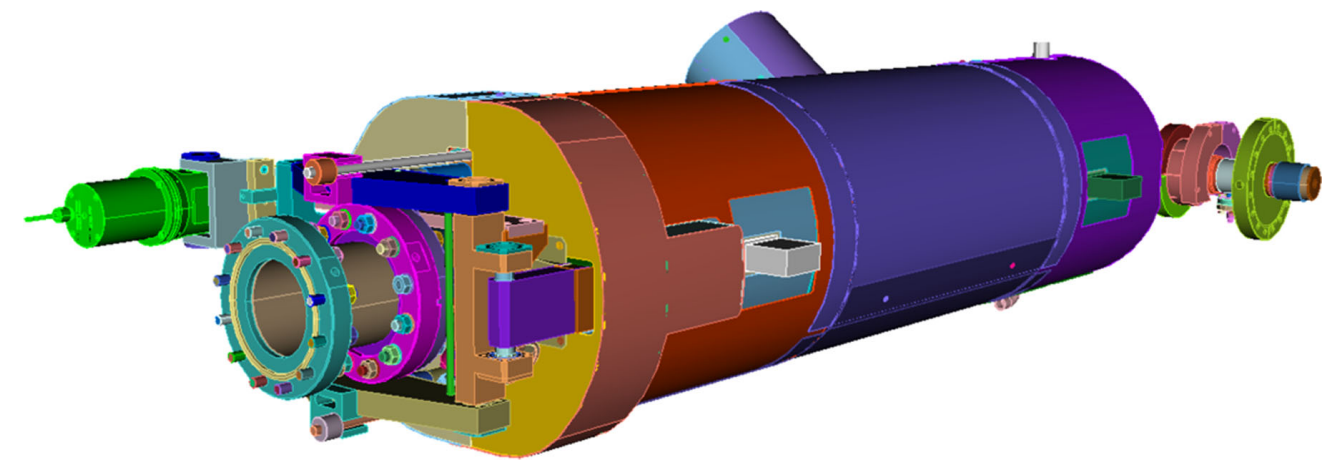

FIG. 1. Illustration of the LCLS-II dressed cavity showing the outer helium vessel wrapped with magnetic shielding, the fundamental power coupler (on the right) and the tuner (on the left) that has both coarse (motor) and fine (piezoactuator) axial positioning control. 

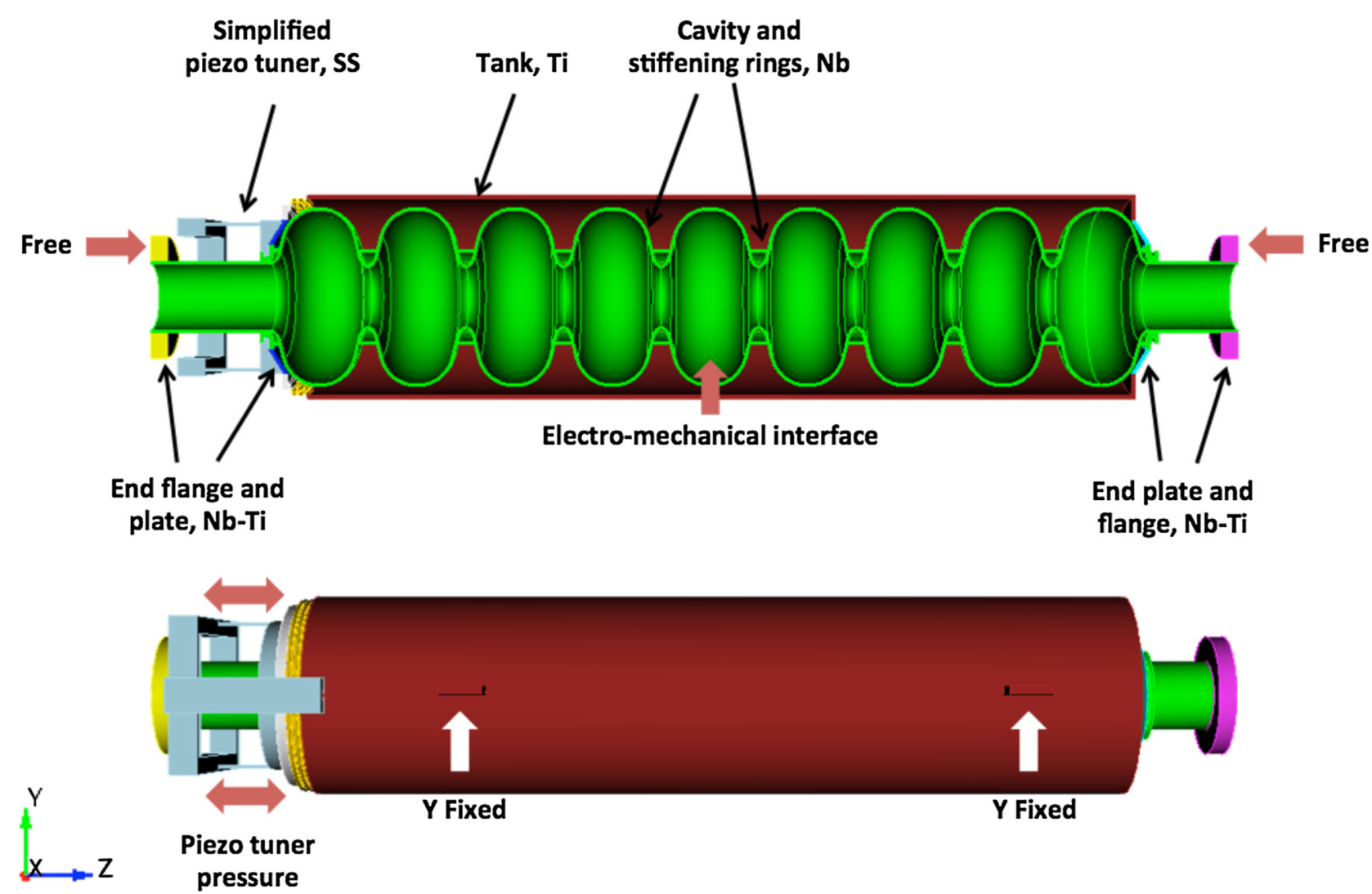

FIG. 2. The vertical cross section (top) and full (bottom) simulation model of the LCLS-II cavity including the electromechanical interface, boundary conditions, materials and the simplified piezotuner model.

this constraint bounded by the two extremes discussed above.

The mechanical properties of the dressed cavity materials as measured at FNAL [32] at $2 \mathrm{~K}$ are listed in Table I and used for our model. The most important of these is the Young's modulus of $\mathrm{Nb}$, which others have measured in range of 105-124 GPa [33]. For the following Lorentz detuning factor and piezotransfer function simulations, we also consider a value of $88.5 \mathrm{GPa}$ as found in stress tests of single-cell 1.5 GHz cavities [33].

To tune the LCLS-II cavity, electromechanical and piezoactuators will be used [4]. The former will be used for slow/coarse adjustments to initially set the frequency and to compensate for long term drifts. In this paper we consider only the piezoelectric actuators, which would be

TABLE I. Mechanical properties of the dressed cavity materials.

\begin{tabular}{lccc}
\hline \hline Material & $\begin{array}{c}\text { Density } \\
{\left[\mathrm{kg} / \mathrm{m}^{3}\right]}\end{array}$ & $\begin{array}{c}\text { Poisson } \\
\text { ratio }\end{array}$ & $\begin{array}{c}\text { Young's modulus } \\
{[\mathrm{GPa}]}\end{array}$ \\
\hline $\mathrm{Nb}$ & 8700 & 0.38 & 118 \\
$\mathrm{Nb}-\mathrm{Ti}$ & 5700 & 0.33 & 68 \\
$\mathrm{Ti}$ & 4540 & 0.37 & 117 \\
$\mathrm{SS}$ & 8000 & 0.29 & 193 \\
\hline \hline
\end{tabular}

used to compensate microphonics, if needed, while the cavity gradient is held nearly constant by the LLRF feedback system.

In Fig. 3, a corresponding longitudinal spring model of the dressed cavity is shown to illustrate the forces at play assuming the He vessel is free to move axially. In the 3D model, we simulate the tuner arms as a stainless steel frame

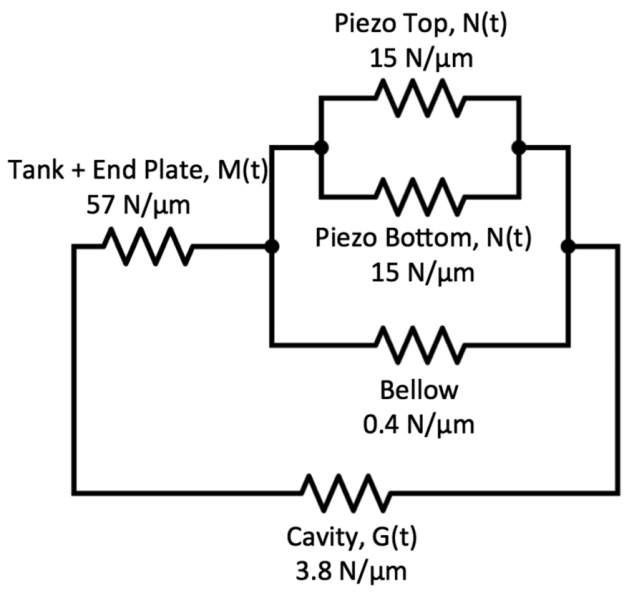

FIG. 3. Spring model of the cavity in a helium tank equipped with bellows and piezotuner. 


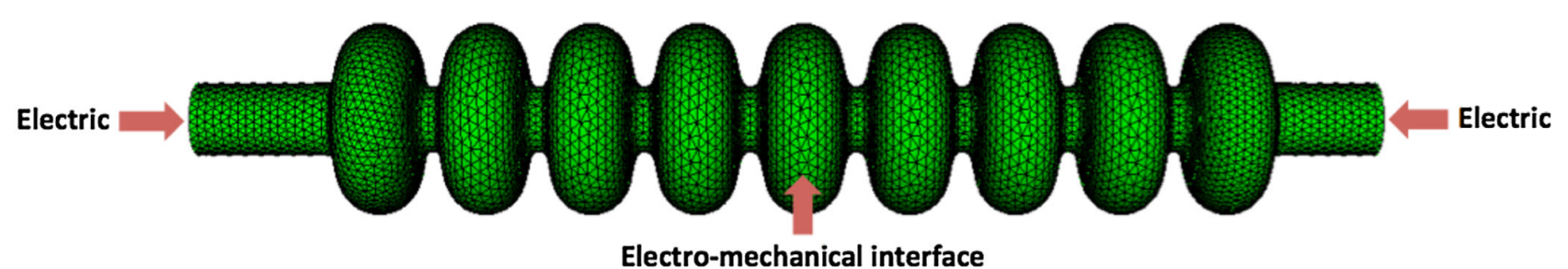

FIG. 4. Vacuum model of the TESLA cavity meshed with curved tetrahedral elements. Also shown are the boundary conditions and the electromechanical interface.

and the piezoactuators are modeled as two stainless steel rods (indicated as "Simplified piezotuner" in Fig. 2) with an effective stiffness of $30 \mathrm{~N} / \mu \mathrm{m}$ [4], i.e., $15 \mathrm{~N} / \mu \mathrm{m}$ per rod. Estimates of the other stiffnesses are: tank plus end plate $=57 \mathrm{~N} / \mu \mathrm{m}$, cavity $=3.8 \mathrm{~N} / \mu \mathrm{m}$ and the bellows between the beam pipe and $\mathrm{He}$ vessel $=0.4 \mathrm{~N} / \mu \mathrm{m}$ [34]. In our study, the only simplification made is for the tuner, the other elements are taken into account directly through the corresponding 3D models.

\section{Vacuum model of the LCLS-II TESLA cavity}

For the electromagnetic calculations, the vacuum model of the TESLA cavity was used as shown in Fig. 4, the surface of which is the electromechanical interface. To better approximate the complicated geometry, curved tetrahedral elements were used to mesh both the structural and vacuum models using CUBIT [35].

With this model, the ACE3P rf eigenmode solver [36] was used to determine the field distribution and the resonant frequency. In Fig. 5, the complex magnitudes of the electric and magnetic fields are illustrated for the fundamental $1.3 \mathrm{GHz} \pi$-mode with the nominal geometry.

For the mechanical simulations discussed below, the deformation of the structural mesh is coupled to the vacuum one through the electromechanical interface, that is, the internal cavity surface shown in Fig. 2 and the external surface of the corresponding vacuum model shown in Fig. 4. Subsequently, the cavity detuning was calculated either by numerically integrating Eq. (11) or by computing the fundamental mode frequency for the deformed vacuum mesh using the ACE3P electromagnetic eigensolver.

\section{Electromechanical characterization of the mechanical eigenmodes}

Based on the structural and vacuum FEM models of the dressed cavity we calculated the eigenfrequencies and the normalized detuning amplitude $\frac{\Delta f_{\mu}}{\max (|\vec{u}|)}$ for the mechanical modes up to $1 \mathrm{kHz}$ assuming the He vessel motion is not constrained in $X$ and $Z$. The results are shown in Fig. 6 .

As expected, the longitudinal cavity modes produce the largest detuning per unit of maximum deformation. As an illustration, Fig. 7 shows snapshots of the structural displacements (also color coded and exaggerated in magnitude for clarity) for the lowest frequency transverse mode (at $56 \mathrm{~Hz}$ ) and the lowest two longitudinal modes (at $238 \mathrm{~Hz}$ and $300 \mathrm{~Hz}$ ). These modes detune the cavity by $0.12,160.3$ and $516.8 \mathrm{~Hz} / \mu \mathrm{m}$, respectively. For the $238 \mathrm{~Hz}$ mode the He vessel moves in opposition to the net cavity displacement, preserving the center of the mass, for the $300 \mathrm{~Hz}$ mode, the He vessel is basically stationary but is deformed to have an elliptical cross section.
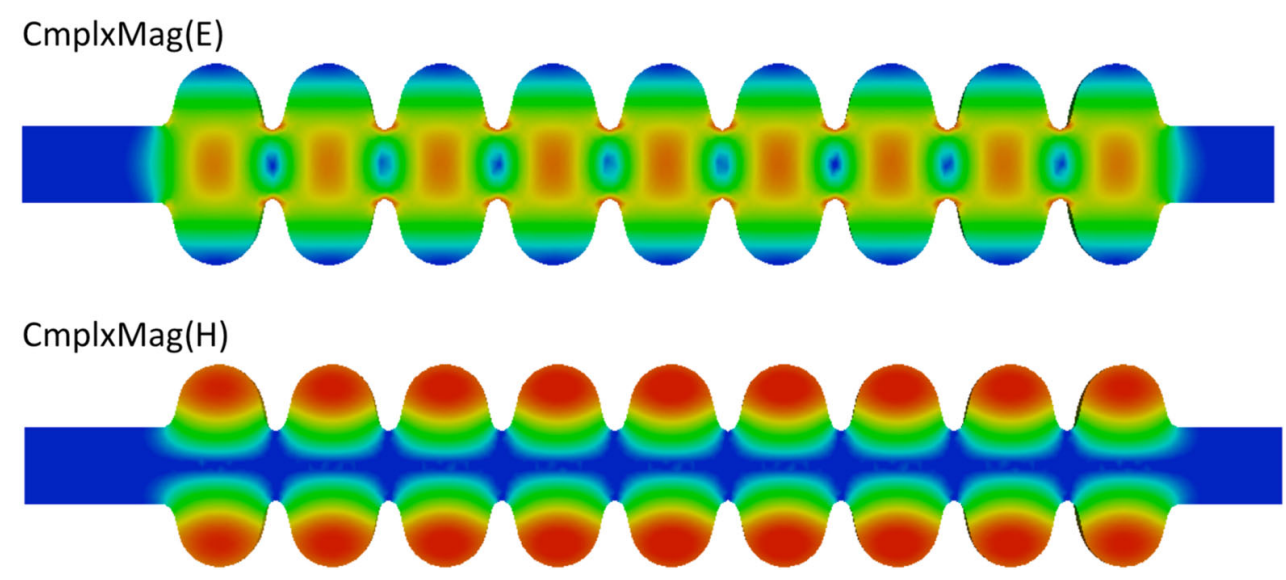

FIG. 5. Complex magnitudes of electric (top) and magnetic (bottom) fields (a.u.) for the $1.3 \mathrm{GHz}$ acceleration mode color coded on a linear scale. 


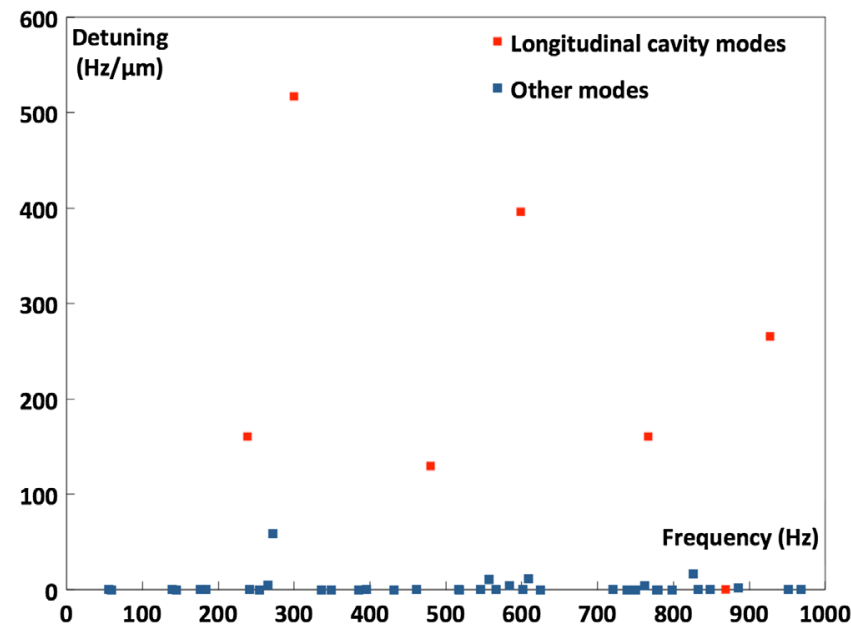

FIG. 6. Cavity detuning normalized to the maximum structural displacement as a function of the mechanical eigenmode frequency. Longitudinal cavity modes are highlighted in red.

For these simulations, curved tetrahedral meshes of up to 3 million elements (16 million degrees of freedom) and vacuum meshes of up to 600 thousand elements (4 million degrees of freedom) were used. It took 2 hours of execution time on 320 cores of the Cori supercomputer [31] to process 50 modes, the total memory used was about $800 \mathrm{~GB}$.

\section{CAVITY RESPONSE TO A STATIC LORENTZ FORCE AND PIEZOLOAD}

\section{A. Static cavity response}

In the cavity, the rf fields exert a pressure, $\vec{t}_{0}$, on the surfaces that is proportional to the square of the field amplitude, that is,

$$
\vec{t}_{0}=\frac{1}{4}\left(\mu|\vec{H}|^{2}-\varepsilon|\vec{E}|^{2}\right) \vec{n} .
$$

This vector points inward or outward from the surface depending on the field pattern and the pressure causes a shift of the resonant frequency known as Lorentz force detuning. Based on the field profile shown in Fig. 5, the electromagnetic pressure was calculated on the walls of the cavity and is illustrated in Fig. 8.

In steady-state rf operation, this load deforms the cavity walls by amounts governed by Eqs. (15)-(17). Employing the boundary conditions, Eqs. (19) and (20), the following weak FEM formulation can be easily derived for the test function $\vec{v}$ :

$$
\int_{\mathrm{V}_{S}}\left[\mu\left(\nabla \vec{u}+\nabla \vec{u}^{T}\right)+\lambda(\nabla \cdot \vec{u}) I\right] \cdot \nabla \vec{v} d \mathrm{~V}_{S}=\int_{\Gamma_{N}}\left(\vec{v} \cdot \vec{t}_{0}\right) d \Gamma_{N} .
$$

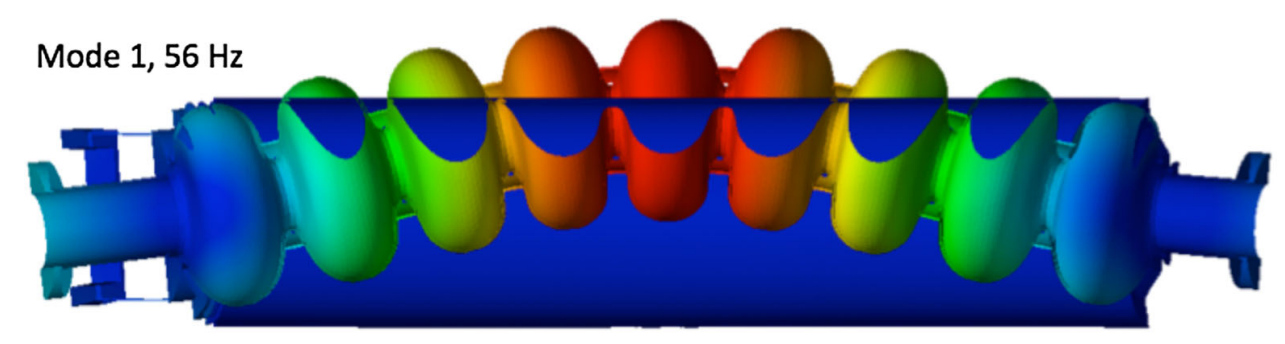

Mode 7, $238 \mathrm{~Hz}$

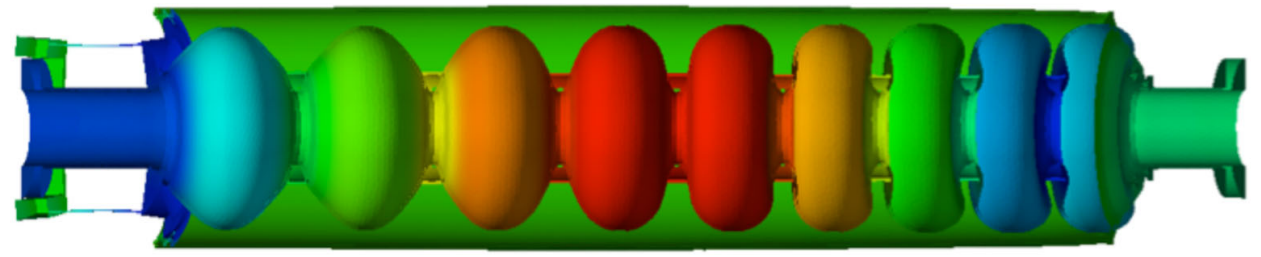

Mode $12,300 \mathrm{~Hz}$

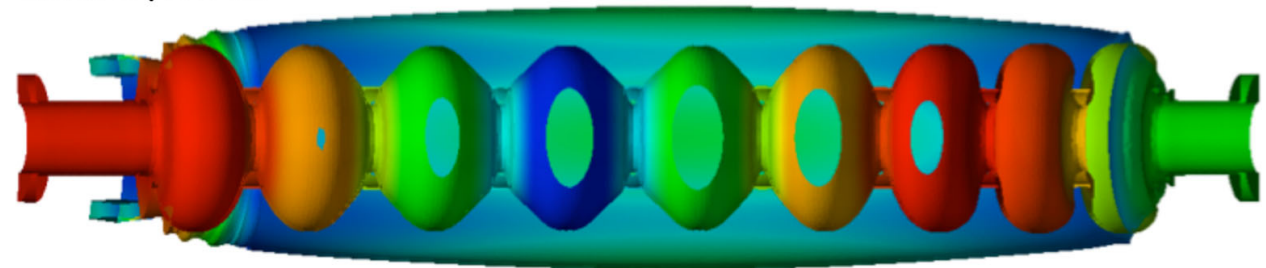

FIG. 7. Illustrations of the structural displacements for three selected modes. The magnitudes of the displacements (a.u.) are color coded on a linear scale. 


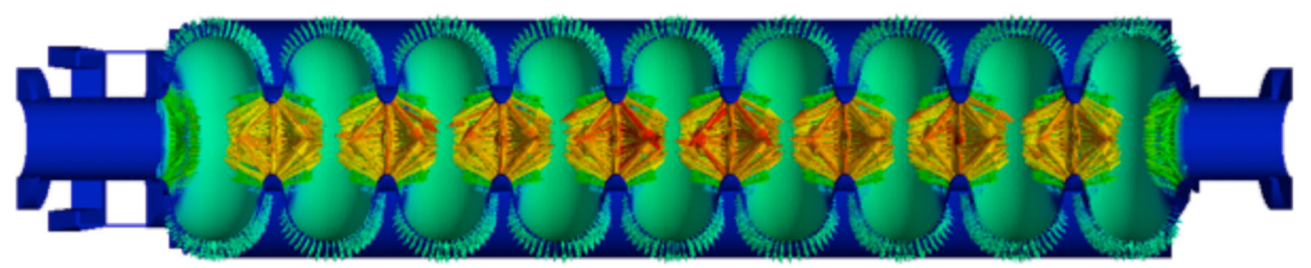

FIG. 8. Direction and magnitude of the electromagnetic pressure (a.u.) color coded on a linear scale for the fundamental $1.3 \mathrm{GHz}$ accelerator mode.

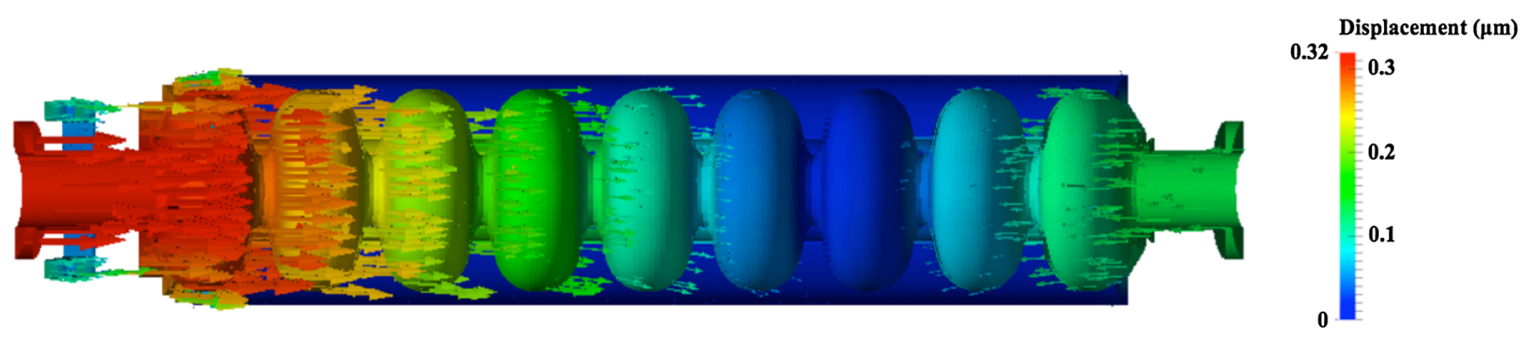

FIG. 9. Direction and magnitude of the structural displacements due to a static Lorentz force.

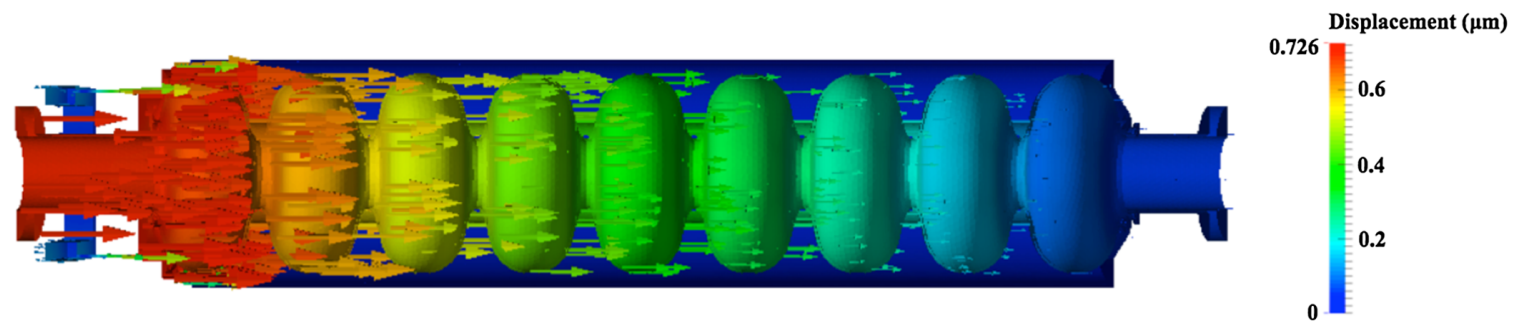

FIG. 10. Direction and magnitude of the structural displacements due to a static piezoload.

Discretizing Eqs. (23) [29], the following linear equation is obtained:

$$
K \hat{u}=b,
$$

where $b$ is determined by the electromagnetic pressure or external load.

For an acceleration gradient of $G_{0}=16 \mathrm{MV} / \mathrm{m}$, we calculated the resulting structural deformation with ACE3P. The cavity deformation was also simulated for a static piezoload of $1 \mathrm{MPa}$. The structural displacements due to the static Lorentz force (LF) and static piezoload (PZ) are shown in Figs. 9 and 10, respectively.

The static piezoload results in a frequency shift of $\Delta f_{P Z}=$ $-219.03 \mathrm{~Hz}$ and the Lorenz force deformation of the cavity leads to a frequency shift of $\Delta f_{\mathrm{LF}}=-240.58 \mathrm{~Hz}$. The resulting Lorentz force detuning coefficient, defined as $k_{L F}=$ $2 \pi \Delta f_{L F} / G_{0}^{2}$, is compared in Table II to values measured at FNAL for the first two LCLS-II cryomodules [37].

For the simulations, assuming a Young's modulus of 88.5 GPa as in [33] yields a better match to the data. The finite-element meshes used for this computation were identical to the ones developed for the eigenmodes in Sec. III. The total runtime was about 30 minutes on 320 cores on the Cori supercomputer and the total memory used was about 1 TB.

\section{B. Mechanical eigenmode decomposition}

Based on Eqs. (6) and (7), a parallel decomposition tool was developed in ACE3P to compute the 3D overlap of the

TABLE II. Lorentz force detuning coefficients (mean values and standard deviation, $\sigma$ ) as measured at FNAL for the eight cavities in cryomodules $\mathrm{CM} 01$ and $\mathrm{CM} 02$, and simulated with ACE3P assuming two values for the Young's modulus of niobium, $E^{N b}$.

\begin{tabular}{lcc}
\hline \hline & $\begin{array}{c}k_{L F} / 2 \pi \\
{\left[\mathrm{Hz} /(\mathrm{MV} / \mathrm{m})^{2}\right]}\end{array}$ & $\begin{array}{c}\sigma / 2 \pi \\
{\left[\mathrm{Hz} /(\mathrm{MV} / \mathrm{m})^{2}\right]}\end{array}$ \\
\hline FNAL measurement & -1.05 & 0.14 \\
CM01 & & 0.07 \\
FNAL measurement & -1.09 & \\
$\quad$ CM02 & & \\
Simulation with & -1.10 & \\
$E^{N b}=88.5 \mathrm{GPa}$ & & \\
Simulation with & -0.94 & \\
$E^{N b}=118 \mathrm{GPa}$ & & \\
\hline \hline
\end{tabular}


TABLE III. Decomposition of cavity detuning from the static Lorentz force and piezoload. Only the contributing modes are shown, the longitudinal cavity modes are highlighted in bold.

\begin{tabular}{lccc}
\hline \hline Mode \# & Frequency $(\mathrm{Hz})$ & $\Delta f_{L F \mu}(\mathrm{Hz})$ & $\Delta f_{P Z \mu}(\mathrm{Hz})$ \\
\hline $\mathbf{7}$ & $\mathbf{2 3 8}$ & $-\mathbf{1 3 . 7}$ & $\mathbf{- 4 2 . 8}$ \\
11 & 272 & -2.4 & -3.4 \\
$\mathbf{1 2}$ & $\mathbf{3 0 0}$ & $\mathbf{- 9 7 . 5}$ & $\mathbf{- 1 4 4 . 8}$ \\
$\mathbf{1 9}$ & $\mathbf{4 8 0}$ & $-\mathbf{4 . 6}$ & $\mathbf{- 2 1 . 8}$ \\
23 & 557 & 0.0 & 0.5 \\
$\mathbf{2 6}$ & $\mathbf{5 9 9}$ & $-\mathbf{2 4 . 4}$ & $\mathbf{- 1 0 . 2}$ \\
$\mathbf{3 4}$ & $\mathbf{7 6 7}$ & $-\mathbf{1 . 0}$ & $\mathbf{1 2 . 5}$ \\
38 & 826 & -0.1 & -1.6 \\
$\mathbf{4 3}$ & $\mathbf{9 2 7}$ & $-\mathbf{8 . 5}$ & $-\mathbf{6 . 5}$ \\
47 & 1025 & -0.1 & -0.6 \\
Sum over the first 50 modes & -152.5 & -218.8 \\
Static frequency shift & -240.6 & -219.0 \\
\hline \hline
\end{tabular}

mode spatial patterns and the static displacements due to various forces. To calculate the spatial overlap amplitudes $q_{\mu}^{0}$, we used the same structural meshes for both the eigenmode and static simulations, employing 3D Gaussian quadratures for evaluating the integral from (6).

The contribution of a particular mechanical mode $\mu$ to static detuning, $\Delta f_{0 \mu}$, is calculated as $q_{\mu}^{0} * \Delta f_{\mu}$, where $\Delta f_{\mu}$ is the detuning caused due to mechanical mode $\mu$ determined in Sec. III D. The Lorentz coefficients and coefficients that correspond to the piezoload in (4) are determined as $k_{\mu}=2 \pi \Delta f_{L F \mu} / G_{0}^{2}$ and $n_{\mu}=2 \pi \Delta f_{P Z \mu} / N_{P Z}$, respectively.

In Table III the results of the decomposition are presented for the static Lorentz force and static piezoload. The total decomposition runtime was about 15 minutes on 320 cores on the Cori supercomputer, the total memory used was about 1 TB.

The frequency shifts $\Delta f_{L F \mu}$ are negative for all the modes as predicted by the analytical models in $[6,16]$, while $\Delta f_{P Z \mu}$ have different signs as expected [16]. It can also be seen that the static Lorentz force mostly couples to the longitudinal cavity modes, which were shown to have the largest detuning per unit of maximum deformation in Fig. 6. Based on the derived coefficients $k_{\mu}$ and $n_{\mu}$, the dynamic frequency shifts $\Delta \omega_{\mu}(t)$ can now be calculated using Eq. (13) as a solution to Eq. (12) for a specific forcing term.

The 36\% discrepancy between the static frequency shift and the summation over the first 50 modes (up to $\sim 1.1 \mathrm{kHz}$ ) for the Lorentz force indicates that there are also contributions from modes at higher frequencies. The discrepancy is significantly smaller for the piezoactuator load as it is a more 1D like deformation than the essentially 2D Lorentz force, as shown in Fig. 8.

The piezoactuator load couples to the same modes as the Lorentz force, although with different strengths, so using the piezoactuator to compensate fast Lorentz force detuning is best done on a short time scale (i.e., less than the mode oscillation periods), as is the case for short pulse operation. For slow changes of the gradient (i.e., greater than mode damping times), having the piezoforce track the square of the gradient is also effective. The difference in the detuning response from these two forces has been confirmed experimentally [16].

\section{CAVITY RESPONSE TO A HARMONIC PIEZOFORCE}

\section{A. Cavity harmonic response}

To calculate the cavity response to various excitations, a massively parallel harmonic response solver was developed [38]. This solver is based on the higher-order FEM to solve Eqs. (15)-(17) in the case of a harmonically varying displacement, Eq. (19), or load, Eq. (20). In this case the weak form of the governing equations becomes

$$
\begin{gathered}
\int_{\mathrm{V}_{S}}\left[\mu\left(\nabla \vec{u}+\nabla \vec{u}^{T}\right)+\lambda(\nabla \cdot \vec{u}) I\right] \cdot \nabla \vec{v} d \mathrm{~V}_{S} \\
=\Omega^{2} \int_{\mathrm{V}_{S}} \rho(\vec{u} \cdot \vec{v}) d \mathrm{~V}_{S}+\int_{\Gamma_{N}}\left(\vec{v} \cdot \vec{t}_{0}\right) d \Gamma_{N}
\end{gathered}
$$

and the corresponding linearized system can be written as

$$
\left(K-\Omega^{2} M\right) \hat{u}=b .
$$

To take into account damping we used the proportional Rayleigh model [28] incorporating the damping matrix $C$ in Eq. (26) as

$$
\begin{gathered}
\left(K-\Omega^{2} M+i \Omega C\right) \hat{u}=b, \\
C=\alpha M+\beta K, \\
\alpha=\frac{2 \zeta \Omega_{m} \Omega_{n}}{\Omega_{m}+\Omega_{n}}, \\
\beta=\frac{2 \zeta}{\Omega_{m}+\Omega_{n}},
\end{gathered}
$$

where $\alpha$ and $\beta$ are the mass and stiffness damping coefficients, respectively, $\Omega_{m}$ and $\Omega_{n}$ are the frequencies of the lowest and the highest contributing resonant modes, $\zeta$ is their critical damping ratio. This formalism ensures that the damping is roughly constant between $\Omega_{m}$ and $\Omega_{n}$. Unfortunately, there is no reliable way to determine $\zeta$ numerically and there are no definitive measurements for TESLA-like cavities, so the critical damping was assumed to be $0.3 \%$, the value experimentally determined during the development of similar accelerating structures [12].

The cavity structural response was simulated for a harmonically varying piezoload with a magnitude $N_{P Z}=$ $1 \mathrm{MPa}$ for frequencies $\Phi_{P Z}$ up to $1 \mathrm{kHz}$, and at each frequency the corresponding detuning magnitude was calculated. For these simulations, the $\mathrm{He}$ vessel was 


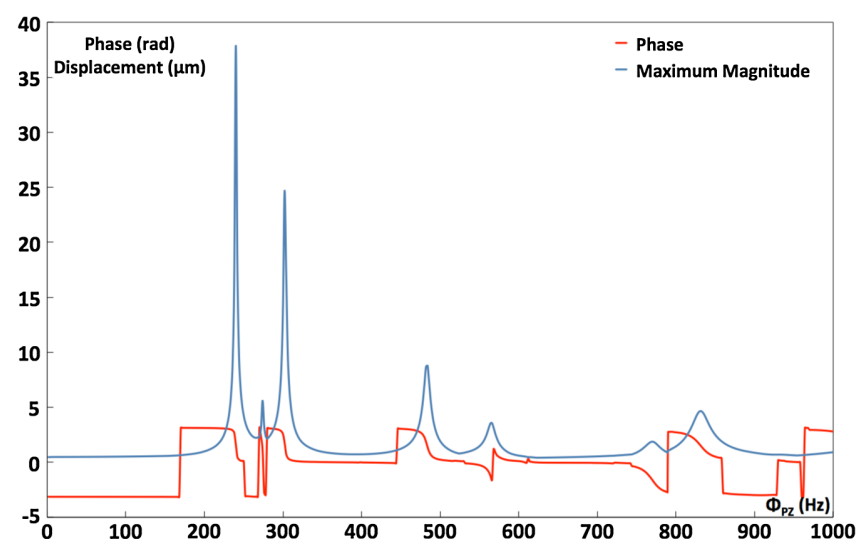

FIG. 11. Maximum displacement magnitude (blue) and the corresponding phase of the load (red) as a function of the piezoactuator frequency.

modeled as if were not constrained axially. In Fig. 11, the maximum displacement magnitude over the cavity and the corresponding phase of the load are plotted as a function of the piezoactuator frequency.

In Fig. 12, the piezo-to-cavity transfer function $\Delta f_{P Z}\left(\Phi_{P Z}\right)$ calculated directly from the structural deformations at each frequency is compared to that computed by summing up the modal responses, i.e., Eq. (13), for the mechanical modes listed in Table III. Since the mechanical eigensolver does not account for damping, the damping ratios $\zeta_{\mu}$ in Eq. (13) were adjusted to match the amplitudes of the simulated harmonic response at the resonant frequencies.

The two methods produce similar results up to about $500 \mathrm{~Hz}$. As the interference between the modes becomes more complicated and the contribution of modes above $1 \mathrm{kHz}$ becomes non-negligible, some discrepancy between the two methods appears. It means that if all the required modes are taken into account with the correct damping information, the transfer function calculation can be

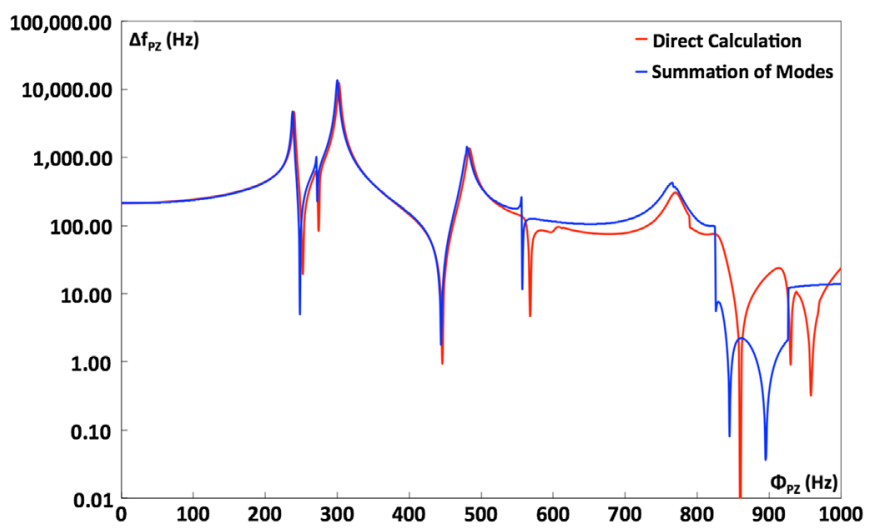

FIG. 12. Detuning magnitude as a function of the piezoactuator frequency $\Phi_{P Z}$ calculated directly from the structural deformations at each frequency (red) and by summing the modal response functions (blue). reduced to the eigenmode and the corresponding decomposition calculations as discussed in Secs. III and IV.

\section{B. Comparison with piezotransfer function measurements}

As part of cavity and cryomodule testing for LCLS-II, the piezo-to-cavity transfer function is measured by driving the piezoactuator sinusoidally with a fixed peak voltage while stepping the frequency of the drive signal and measuring the resulting cavity detuning. Figure 13 shows two examples of such measurements, one taken for cavity 2 of CM02 at FNAL [37], and one of a cavity in a horizontal test stand at Cornell $[39,40]$. The two results agree fairly well except in the 100-200 Hz range, where the Cornell results show more features (the cavity axial constraints in this case likely differed from that in a LCLS-II cryomodule). Also shown in Fig. 13 are the simulation results for two cases: assuming the He vessel is fixed in $\mathrm{Y}$ only and fixed in YZ. Both assume $E^{N b}=88.5 \mathrm{GPa}$ for the cavity, which provided a better match to the Lorentz force

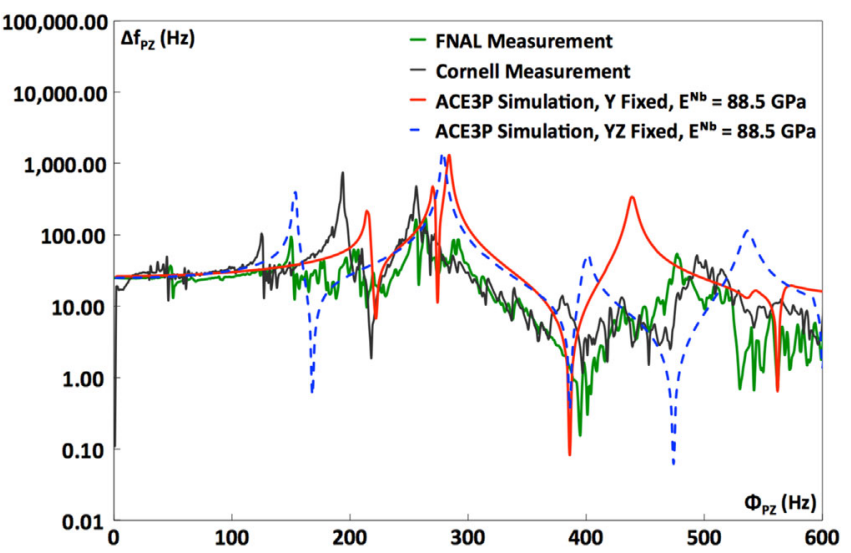

FIG. 13. Measurements and simulations of cavity detuning as a function of the piezoactuator frequency-the curves are described in the text. 
detuning data as noted above. The simulations are normalized to match the data at low frequency.

One can see that the axial constraint has little effect on the simulated response of the second longitudinal mode near $280 \mathrm{~Hz}$ in which the $\mathrm{He}$ vessel remains basically stationary as noted earlier. It does have a large effect on the frequency on the lowest longitudinal mode where the $\mathrm{He}$ vessel increases the mass of the system in motion if unconstrained.

While there are general features that are common to the simulations and data, the measured detuning response is much more nuanced. The peaks at $260 \mathrm{~Hz}$ in the data are likely the second longitudinal mode - the phase of the detuning measured at FNAL shows a clear jump by 180 degrees at this frequency [37]. The response of the first longitudinal mode seems washed out in the FNAL data, but appears in the Cornell data about $30 \mathrm{~Hz}$ below the Y-fixed simulation peak for this mode. This frequency shift is similar to that for the second longitudinal mode, so if $E^{N b}$ was decreased, both peaks could be made to match better, although the higher frequency match would be worse.

Undoubtedly, the cavity detuning response is more complicated than that represented by our dressed cavity model. Several factors likely contribute: knowledge of the actual material properties, not having well defined boundary conditions and not fully modeling the system (the effects of the power couplers, liquid $\mathrm{He}$, connecting bellows and the tuner substructure are not included). Also, the data has "feedthrough" from detuning unrelated to the piezomotion and the piezoforce may be more complicated than that modeled. The effect of the coupler and multicavity modes have been recently studied with ACE3P [41] but the discussion is beyond the scope of this paper.

For the harmonic response calculation, a structural mesh consisting of 600 thousand curved tetrahedral elements (3.7 million degrees of freedom) was used with a corresponding vacuum mesh with 200 thousand elements. The total runtime for 1,000 frequency steps was about 5 hours on 128 cores on the Cori system, the total memory used was about 200 GB.

\section{CONCLUSION}

New simulation capabilities for 3D multiphysics modeling of SRF cavities were used to study the electromechanical characteristics of the LCLS-II TESLA-type dressed cavity. The simulation results are in fair agreement with measurements of Lorentz force detuning and detuning from sinusoidal piezoforces, although the model clearly has limitations in representing the complicated cavity support system and its connections to other elements in the cryomodule. Nonetheless, this study provides information that is useful for designing a resonance control system for the LCLS-II cavity.
The parallel finite-element multiphysics simulation suite ACE3P also allows for the rf, mechanical and thermal studies of more complex models, for instance, a string of cavities in a cryomodule [42-44]. In this case, special attention should be paid to the mesh as well as to the simulation settings that depend on the particular problem size and the amount of memory available per a computing node. By doing the domain decomposition, fairly large problems (hundreds millions degrees of freedom) can be solved with ACE3P being only limited by the scalability of the available linear solvers.

The analysis presented in this work would not be feasible using other commercial software running on conventional desktops with limited computing resources. The parallel modeling capabilities developed for large-scale modeling of the LCLS-II SRF cavity systems are equally applicable to a wide range of multiphysics SRF applications in other accelerator projects, providing the accelerator community the needed tools to solve challenging problems at a simulation scale not possible before.

\section{ACKNOWLEDGMENTS}

The work was supported by U.S. Department of Energy under Contract No. DE-AC02-76SF00515. This research used resources of the National Energy Research Scientific Computing Center, which is supported by the Office of Science of the U.S. DOE, Contract No. DE-AC0205CH11231.

[1] A. W. Chao, H. O. Moser, and Z. Zhao, Accelerator Physics, Technology and Applications (World Scientific, Singapore, 2004).

[2] H. Padamsee, The science and technology of superconducting cavities for particle accelerators, Supercond. Sci. Technol. 14, R28 (2001).

[3] Y. Pischalnikov, E. Borissov, I. Gonin, J. Holzbauer, T. Khabiboulline, W. Schappert, S. Smith, and J. C. Yun, Design and test of compact tuner for narrow bandwidth SRF cavities, in Proceedings of IPAC2015, Richmond, VA, 2015.

[4] E. Borissov, I. Gonin, Y. Pischalnikov, and W. Schappert, LCLS II cavity tuner, LCLS II Dressed Cavity Review, 2014.

[5] L. R. Doolittle et al., LLRF control of high $\mathrm{Q}_{L}$ cavities for the LCLS-II, in Proceedings of IPAC2016, Busan, Korea, pp. 2765-2767.

[6] C. Serrano, L. R. Doolittle, G. Huang, and P. J. Emma, Report No. LCLSII-TN-17-06, 2017.

[7] J. Delayen, Ponderomotive instabilities and microphonicsA tutorial, Physica C (Amsterdam) 441C, 1 (2006).

[8] M. M. Karliner, V.E. Shapiro, and I. A. Shekhtman, Instability in the walls of a cavity due to ponderomotive forces of the electromagnetic field, Sov. Phys. Tech. Phys. 11, 1501 (1967). 
[9] D. Schulze, Ponderomotive stability of rf resonators and resonator control systems, in Physics. 1971, University of Karlsruhe: Karlsruhe, Germany (ANL Translation 944).

[10] J. C. Slater, Microwave electronics, Rev. Mod. Phys. 18, 441 (1946).

[11] Z. A. Conway, Ph.D. thesis, University of Illinois at Urbana-Champaign, 2007.

[12] A. Kumar, A. R. Jana, and V. Kumar, A study of dynamic Lorentz force detuning of $650 \mathrm{MHz} \beta_{g}=0.9$ superconducting radiofrequency cavity, Nucl. Instrum. Methods Phys. Res., Sect. A 750, 69 (2014).

[13] G. Devanz, M. Luong, and A. Mosnier, Numerical simulations of dynamic Lorentz detuning of SC cavities, in Proceedings of the 8th European Particle Accelerator Conference, Paris, 2002 (EPS-IGA and CERN, Geneva, 2002), pp. 2220-2222.

[14] V. Goryashko, FREIA Report No. 2014/02.

[15] R. Mitchell et al., Lorentz force detuning analysis of the Spallation Neutron Source (SNS) accelerating cavities, in Proceedings of the 10th Workshop on rf Superconductivity, Tsukuba, Japan, 2001, pp. 236-242.

[16] M. Doleans, Ph.D. thesis, Paris 7 University, 2003 (https:// web.archive.org/web/20111024205820/http://www.sns .gov/APGroup/Papers/DoleansThesis.pdf).

[17] ANSYs, http://www.ansys.com/.

[18] CST, http://www.cst.com/.

[19] COMSOL, http://www.comsol.com/.

[20] ACE3P SIMULATION SUITE, SLAC National Accelerator Laboratory, http://www-group.slac.stanford.edu/acd/.

[21] K. Ko et al., Advances in parallel electromagnetic codes for accelerator science and development, in Proceedings of the 25th International Linear Accelerator Conference, LINAC-2010, Tsukuba, Japan (KEK, Tsukuba, Japan, 2010), pp. 1028-1032.

[22] L. Xiao et al., TTF3 power coupler thermal analysis for LCLS-II cw operation, in Proceedings of IPAC15, Richmond, VA (2015).

[23] T. Luo et al., Thermal and Lorentz force analysis of beryllium windows for a rectilinear muon cooling channel, in Proceedings of IPAC2015, Richmond, VA (2015), p. 3.

[24] S. Verdú-Andrés et al., Frequency tuning for a DQW crab cavity, in Proceedings of IPAC16, Busan, Korea (2016), pp. 2357-2359.

[25] Linac Coherent Light Source II, Official web page, https:// portal.slac.stanford.edu/sites/lcls_public/lcls_ii/Pages/default .aspx.

[26] B. Aune et al. Superconducting TESLA cavities, Phys. Rev. ST Accel. Beams 3, 092001 (2000).
[27] https://en.wikipedia.org/wiki/Harmonic_oscillator\#Steady -state_solution.

[28] S. Adhikari, Ph.D. thesis, Cambridge University, UK, 2000.

[29] O. C. Zienkiewicz and R. L. Taylor, The Finite Element Method for Solid and Structural Mechanics (ButterworthHeinemann, Oxford, 2005).

[30] O. Kononenko et al., Report No. SLAC-PUB-15976, 2014.

[31] National Energy Research Scientific Computing Center, Office of Science, and U.S. Department of Energy, http:// www.nersc.gov/.

[32] Material properties for engineering analyses of SRF cavities, Fermilab Specification: ED0371110, 2013.

[33] G. Ciovati, P. Dhakal, J. Matalevich, G. Myneni, A. Schmidt, J. Iversen, A. Matheisen, and W. Singer, Mechanical properties of niobium radio-frequency cavities, Mater. Sci. Eng. A 642, 117 (2015).

[34] S. Tariq, $1.3 \mathrm{GHz}$ cavity/cryostat stiffness model and tuning sensitivity, SRF Engineering Meeting, FNAL, 2006.

[35] The CUBIT Geometry and Mesh Generation Toolkit, https:// cubit.sandia.gov/.

[36] L.-Q. Lee, Z. Li, C. Ng, and K. Ko, Report No. SLACPUB-1352, 2009.

[37] Y. Pischalnikov (private communication).

[38] O. Kononenko, SLAC Report No. SLAC-PUB-16229.

[39] W. Schappert, J. Holzbauer, Yu. Pischalnikov, D. Gonnella, and M. Liepe, Resonance control for narrow-bandwidth, superconducting rf applications, in Proceedings of SRF2015, Whistler, BC, Canada, pp. 828-831.

[40] O. Kononenko, C. Adolphsen, Z. Li, T. Raubenheimer, and C. Rivetta, Electro-mechanical modeling of the LCLS-II superconducting cavities, in Proceedings of LINAC2016, East Lansing, MI.

[41] O. Kononenko, LCLS-II superconducting rf studies, SLAC National Accelerator Laboratory, https://slacspace.slac .stanford.edu/sites/lcls/lcls-2/ap/Pages/scrf.aspx.

[42] C. Ng et al., Design and optimization of large accelerator systems through high-fidelity electromagnetic simulations, J. Phys. Conf. Ser. 125, 012003 (2008).

[43] M. S. Leitner et al., Studies of radiation fields of LCLS-II superconducting radio frequency cavities, Int. J. Mod. Phys. Conf. Ser. 44, 1660209 (2016).

[44] L. Xiao, O. Kononenko, and C.-K. Ng, Simulation of mechanical oscillations in PIP-II cryomodule using ACE3P, in Proceedings of LINAC2016, East Lansing, MI. 